\title{
MRI Features of Aquaporin-4 Antibody-Positive Longitudinally Extensive Transverse Myelitis: Insights into the Diagnosis of Neuromyelitis Optica Spectrum Disorders
}

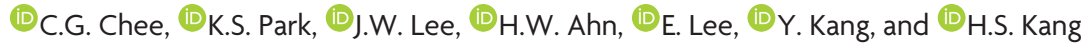

\begin{abstract}
BACKGROUND AND PURPOSE: Longitudinally extensive transverse myelitis is a well-documented spinal manifestation of neuromyelitis optica spectrum disorders, however, other forms of nontumorous myelopathy can also manifest as longitudinally extensive transverse myelitis. Our aim was to evaluate the MR imaging features of aquaporin-4 antibody-positive longitudinally extensive transverse myelitis, which is strongly associated with neuromyelitis optica spectrum disorders.
\end{abstract}

MATERIALS AND METHODS: We evaluated cervicomedullary junction involvement, cord expansion ratios, bright spotty lesions, the number of involved segments, skipped lesions, enhancement patterns, and axial distribution patterns using spinal MR imaging of 41 patients with longitudinally extensive transverse myelitis who underwent aquaporin-4 antibody testing. Univariate logistic regression analysis was performed to identify factors associated with aquaporin-4 antibody seropositivity, which were then used to develop a scoring system for diagnosing aquaporin-4 antibody-positive longitudinally extensive transverse myelitis. Interrater reliability for cord expansion ratio measurement and bright spotty lesions was determined using intraclass correlation coefficients and $\kappa$ values, respectively.

RESULTS: Fifteen patients with longitudinally extensive transverse myelitis were aquaporin-4 antibody-positive. Sex (female), cervicomedullary junction involvement, a cord expansion ratio of $>1.4$, and bright spotty lesions were significantly associated with aquaporin-4 antibody seropositivity. The sensitivity and specificity of the scoring system were $73.3 \%$ and $96.2 \%$, respectively. The interclass correlation value for the cord expansion ratio was 0.78 , and the $\kappa$ value for bright spotty lesions was 0.61 .

CONCLUSIONS: Our scoring system, based on cervicomedullary junction involvement, higher cord expansion ratio, bright spotty lesions, and female sex, can facilitate the timely diagnosis of neuromyelitis optica spectrum disorders.

ABBREVIATIONS: AQP4 = aquaporin-4; LETM = longitudinally extensive transverse myelitis; NMO = neuromyelitis optica; NMOSD = neuromyelitis optica spectrum disorders

ongitudinally extensive transverse myelitis (LETM) is a wellLdocumented spinal manifestation of neuromyelitis optica (NMO) that presents as a hyperintense spinal cord lesion extending over $\geq 3$ vertebral levels on sagittal T2-weighted spinal MR imaging. ${ }^{1} \mathrm{NMO}$ is an inflammatory central nervous system disorder that differs from multiple sclerosis and is associated with the presence of the serum aquaporin-4 (AQP4) antibody as a highly specific feature. ${ }^{1-3}$ It is currently understood as a spectral disorder, and the International Panel for NMO Diagnosis has rede-

Received July 19, 2017; accepted after revision November 29.

From the Departments of Radiology (C.G.C., J.W.L., H.W.A., E.L., Y.K., H.S.K.) and Neurology (K.S.P.), Seoul National University Bundang Hospital, Gyeonggi-do, Korea.

Choong Guen Chee and Kyung Seok Park contributed equally to this work.

Please address correspondence to Joon Woo Lee, MD, PhD, Department of Radiology, Seoul National University Bundang Hospital, 82 Gumi-ro, 173 Beon-gil, Bundang-gu, Seongnam-si, Gyeonggi-do, 13620, Korea; e-mail: joonwoo2@gmail.com

http://dx.doi.org/10.3174/ajnr.A5551 fined the disease entity as NMO spectrum disorders (NMOSD). NMOSD is stratified further according to the presence of the AQP4 antibody. LETM is a diagnostic criterion for $\mathrm{NMOSD}^{4}$; however, other forms of nontumorous myelopathy, such as acute transverse myelitis (idiopathic) or viral myelitis, can also manifest as LETM. There is limited clinical or laboratory information regarding the initial presentation of LETM, which is crucial for diagnosing NMOSD. A long turnaround time (days to weeks) for serologic assay results for the AQP4 antibody further delays the diagnosis of NMOSD. Yet, recent studies demonstrating the beneficial effects of rituximab, plasma exchange, or lymphocytapheresis combined with steroid therapy ${ }^{5-9}$ have emphasized the importance of early diagnosis and timely treatment of NMOSD.

A recent report by Pekcevik et $\mathrm{al}^{10}$ has suggested that characteristic MR imaging findings may help differentiate NMOSD from other pathologies associated with LETM. Yet, the study by Pekcevik et al used criteria-based diagnosis, excluded acute 
transverse myelitis and seronegative NMOSD, and included certain diagnoses based on MR imaging and the clinical setting, such as spinal arteriovenous fistula and acute spinal infarction.

The purpose of our study was to evaluate the imaging features of AQP4-positive LETM, which is highly associated with NMOSD, and to develop a scoring system for diagnosing AQP4-positive LETM on the basis of MR imaging findings. Furthermore, we investigated quantitatively measurable image characteristics and provided interobserver agreement for subjective MR imaging interpretation.

\section{MATERIALS AND METHODS \\ Study Overview}

This retrospective observational study was conducted in an urban teaching hospital. The institutional review board approved the study and waived the requirement for informed consent.

\section{Patients}

We retrospectively reviewed adult (16 years of age or older) patients who had undergone spinal MR imaging between December 2004 and September 2014 and who had initially presented with myelopathy. Patients with MR imaging findings or a clinical course consistent with spinal dural arteriovenous fistula or spinal infarction were excluded. We identified 43 consecutive patients with hyperintense spinal cord lesions extending over $\geq 3$ vertebral levels on sagittal T2-weighted spinal MR imaging. All patients' sera were tested for the presence of the AQP4 antibody at the Weatherall Institute of Molecular Medicine (John Radcliffe Hospital, Oxford, UK) using a cell-based assay. ${ }^{11}$ One patient's MR imaging findings were determined to be artifactual after shortterm follow-up MR imaging. One patient who had undergone the initial MR imaging outside the hospital had not undergone axial imaging. After we excluded these 2 patients, 41 patients were included in our analysis. When available, we also reviewed any brain MR imaging results obtained within an 8-week interval of spinal MR imaging.

\section{MR Image Acquisition}

MR imaging was performed using a 1.5T or 3T scanner (Achieva; Philips Healthcare, Best, the Netherlands) with a 16-channel neurovascular coil for the $3 \mathrm{~T}$ imager and a head neck coil for the $1.5 \mathrm{~T}$ imager. Parameters for individual sequences were as follows: for sagittal T1-weighted TSE images: voxel size $=0.5 \times 0.5 \times 3.0$ $\mathrm{mm}^{3} ; \mathrm{TR}=480-550 \mathrm{~ms} ; \mathrm{TE}=10-15 \mathrm{~ms}$; echo-train length $=6$; slice thickness $=3.0 \mathrm{~mm}$; slice gap $=0.3 \mathrm{~mm}$; NEX $=1-2$; for sagittal T2-weighted TSE images: voxel size $=0.5 \times 0.5 \times 3.0$ $\mathrm{mm}^{3} ; \mathrm{TR}=2700-3500 \mathrm{~ms} ; \mathrm{TE}=120 \mathrm{~ms}$; echo-train length $=$ $28-32$; slice thickness $=3.0 \mathrm{~mm}$; slice gap $=0.3 \mathrm{~mm} ; \mathrm{NEX}=2-3$; for axial T2-weighted TSE images: voxel size $=0.7 \times 0.7 \times 6.0$ $\mathrm{mm}^{3}$; TR $=2700-3500 \mathrm{~ms} ; \mathrm{TE}=120 \mathrm{~ms}$; echo-train length $=$ $25-32$; slice thickness $=6.0 \mathrm{~mm}$; slice gap $=2.0 \mathrm{~mm} ; \mathrm{NEX}=2$. For selected cases, additional thin-section axial TSE images were obtained with the following parameters: voxel size $=0.7 \times 0.7 \times$ $3.0 \mathrm{~mm}^{3} ; \mathrm{TR}=2700-3500 \mathrm{~ms} ; \mathrm{TE}=120 \mathrm{~ms}$; echo-train length $=$ 25-32; slice thickness $=3.0 \mathrm{~mm}$; slice gap $=0.3 \mathrm{~mm}$; NEX $=2$. For patients who underwent the initial MR imaging outside our

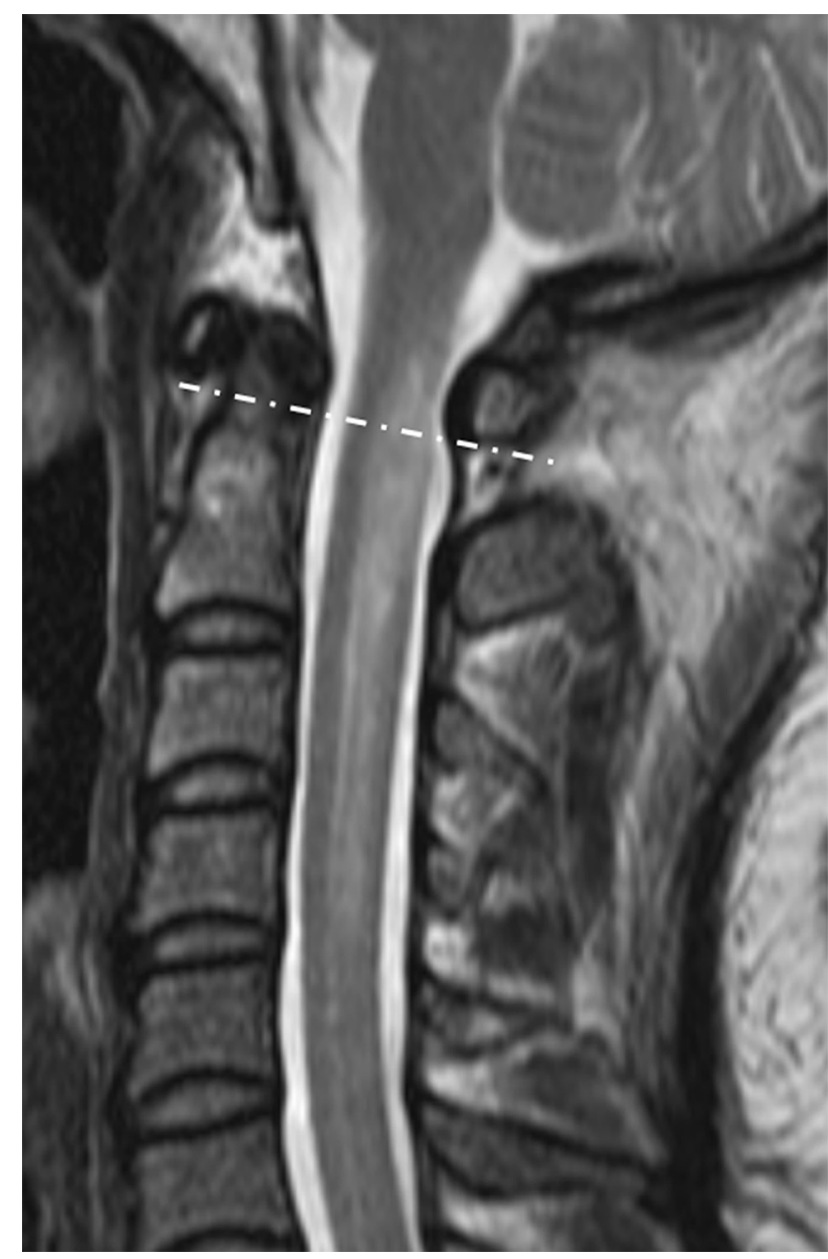

FIG 1. Sagittal T2-weighted spinal MR imaging of a 36-year-old woman with LETM. The lesion extends beyond the imaginary line (dashed line) connecting the inferior cortex of the $\mathrm{Cl}$ anterior and posterior arches. Cervicomedullary junction involvement is present.

hospital, images were imported into our PACS system. These imported MR images had been obtained at diverse hospitals using various MR imaging scanners and different protocols.

\section{MR Image Assessment}

Radiologists were blinded to all patient and clinical data. Cervicomedullary junction involvement was defined as the main or separate lesion involving the uppermost cervical spinal cord at the level of the medulla oblongata. An imaginary line connecting the inferior cortex of the $\mathrm{C} 1$ anterior and posterior arches on sagittal T2WI was used as the lower border (Fig 1), and the pontomedullary junction was used as an upper border. Cord expansion was independently measured on sagittal T2WI by 2 radiologists with 5 and 3 years of experience, respectively. Values were averaged for the analysis. Only MR images obtained during the acute/early stage of disease (within 2 months of onset) ${ }^{12}$ were used for measurement. The ratio of cord expansion was measured as the anteroposterior diameter of the most swollen spinal cord segment to the anteroposterior diameter of the noninvolved spinal cord in the cranial direction on sagittal T2WI (Fig 2). The diameters were measured in millimeters to an accuracy of 1 decimal digit. If LETM extended to the level of the foramen magnum, the ratio was not useful for representing cord expansion due to an anatomic 


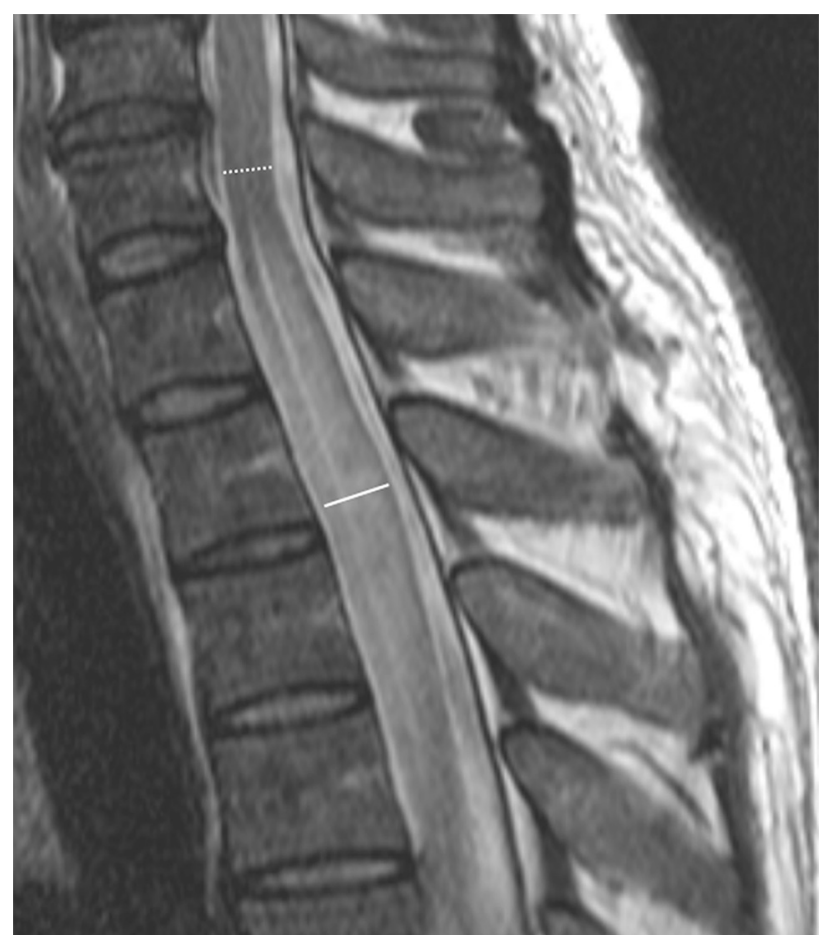

FIG 2. Sagittal T2-weighted spinal MR imaging of a 43-year-old man with LETM. Cord expansion ratio is measured as the anteroposterior diameter of the most swollen spinal cord segment (solid line) to the anteroposterior diameter of the noninvolved spinal cord in the cranial direction (dotted line). Two independent readers measured the cord expansion ratio as 1.65 and 1.71 .

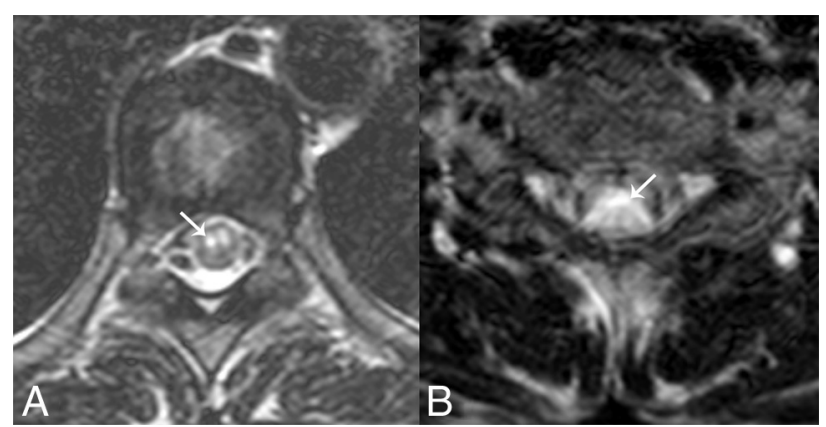

FIG 3. Two examples of bright spotty lesions (white arrows) in axial T2-weighted spinal MR imaging. Two independent readers judged the lesions as positive.

increase in the anteroposterior diameter of the medulla oblongata; thus, ratios were not measured in such cases. Ratio values were dichotomized with a cutoff value to maximize the sum of the sensitivity and specificity.

Bright spotty lesions were defined as lesions that were visually hyperintense or of equal signal intensity to the surrounding CSF on axial T2WI (Fig 3). ${ }^{13}$ Two radiologists independently judged the presence of bright spotty lesions. Suspicious or indeterminate cases were interpreted as having negative findings. In cases of discordance, a consensus reading was performed and used for further statistical analyses. The number of involved segments and the presence of skipped lesions were evaluated. If available, enhancement patterns were assessed by visual inspection and graded as involving $>50 \%$, involving $<50 \%$, or showing no enhancement. Axial distribution was classified as centrally lo- cated, peripherally located, or both. Whether the cord involvement was greater or less than $50 \%$ of the cord in the axial plane was evaluated.

\section{Statistical Analysis}

A univariate logistic regression analysis was performed to identify factors associated with the presence of confirmed AQP4 antibody-positive status. Key factors for the logistic regression analysis were predefined in accordance with previous studies (brain stem involvement, cord expansion, and bright spotty lesions). ${ }^{10,12,14}$ We did not perform a multivariable logistic regression analysis, given the small sample size. Factors that were significantly associated with AQP4 seropositivity were used to generate a scoring system for predicting AQP4 antibody status. Optimal cutoff values that maximized the sum of sensitivity and specificity were used as a threshold. Sensitivity and specificity of the scoring system were measured; interobserver reliability for the measurement of cord expansion was evaluated by using the intraclass correlation coefficient derived from a 2-way mixed-effects model, while interobserver agreement for judging the presence of bright spotty lesions was evaluated using $\kappa$ values. A 2 -sided $P$ value $<$ .05 was considered statistically significant. All statistical analyses were performed using STATA 13.1 (StataCorp, College Station, Texas).

\section{RESULTS}

Of the 41 patients with LETM, 18 were female and 23 were male. The mean age of the patients at the time the spinal MR imaging was obtained was 45 years (age range, 16-72 years). Fifteen patients $(15 / 41,36.6 \%)$ were seropositive for AQP4 antibodies. If we excluded 4 patients who did not undergo tests for an oligoclonal band, all 37 patients were negative for oligoclonal band test. According to the recent diagnostic criteria by Wingerchuk et al, ${ }^{4}$ all 15 patients in the AQP4 antibody-positive group could be diagnosed as having NMOSD, while only 3 of 26 patients (11.5\%) in AQP4 antibody-negative group had it. Seventeen patients underwent $1.5 \mathrm{~T}$ spine MR imaging, and 24 patients underwent $3 \mathrm{~T}$ spine MR imaging. Imaging in 9 patients was performed at outside institutions. The ethnicity of the study population was exclusively Asian. The median time interval between the initial symptom to MR imaging study was 14 days (interquartile range, 3-30 days). Patient characteristics in each group are summarized in Table 1. Thirty-six patients completed brain MR imaging within 2 months after the first attack of myelitis symptoms. Sixteen patients showed suspected demyelinating lesions, and 2 patients had findings suspicious for meningitis. Five patients had a history of optic neuritis preceding the initial symptoms of suspected myelopathy.

Spinal MR imaging findings in the AQP4 antibody-positive and AQP4 antibody-negative groups are shown in Table 2. The cord expansion ratio was measured in 32 patients, excluding 5 patients with dates of onset of $>2$ months before the MR imaging and 4 patients with medulla oblongata involvement. The mean value of the cord expansion ratio was not different whether symptom onset was within 2 months or 15 days (Table 2). Cord expansion ratios were dichotomized using a cutoff value of 1.4. Regression analysis showed that female sex, cervicomedullary junction involvement, a cord expansion ratio of $>1.4$, and the presence of bright 
Table 1: Patient characteristics $(n=41)^{a}$

\begin{tabular}{lcc}
\hline \multicolumn{1}{c}{ Characteristic } & $\begin{array}{c}\text { AQP4 } \\
\text { Antibody-Positive } \\
\text { Group }(\boldsymbol{n}=15)\end{array}$ & $\begin{array}{c}\text { AQP4 } \\
\text { Antibody-Negative } \\
\text { Group }(\boldsymbol{n}=\mathbf{2 6})\end{array}$ \\
\hline Age (mean) (yr) & $48 \pm 13$ & $42 \pm 12$ \\
Female & $13(87 \%)$ & $5(19 \%)$ \\
Ethnicity & Asian (100\%) & Asian (100\%) \\
Time interval from first attack & $10(3-21)$ & $14(5-30)$ \\
$\quad$ to MRI study (median) & & \\
(interquartile range) (days) & $10(67 \%)$ & $14(54 \%)$ \\
1-15 days & $3(20 \%)$ & $6(23 \%)$ \\
$16-30$ days & $1(1 \%)$ & $2(8 \%)$ \\
$31-60$ days & $1(1 \%)$ & $4(15 \%)$ \\
$>60$ days & $8(53 \%)$ & $10(38 \%)$ \\
Brain involvement & $4(27 \%)$ & $1(4 \%)$ \\
Preceding optic neuritis & & \\
\hline
\end{tabular}

${ }^{a}$ Data are number and percentage unless otherwise indicated.

Table 2: Spinal MRI findings in AQP4 antibody-positive and -negative groups ${ }^{\text {a }}$

\begin{tabular}{|c|c|c|}
\hline Imaging Features & $\begin{array}{c}\text { AQP4 } \\
\text { Antibody-Positive } \\
\text { Group ( } n=15)\end{array}$ & $\begin{array}{c}\text { AQP4 } \\
\text { Antibody-Negative } \\
\text { Group }(n=26)\end{array}$ \\
\hline Cervicomedullary junction involvement & $9(60 \%)$ & $6(23 \%)$ \\
\hline Medulla oblongata involvement & $4(27 \%)$ & $1(4 \%)$ \\
\hline Cord expansion ratio (mean) (onset $\leq 2$ months) ${ }^{\mathrm{b}}$ & $1.35 \pm 0.15$ & $1.24 \pm 0.15$ \\
\hline Cord expansion ratio (mean) (onset $\leq 15$ days) ${ }^{c}$ & $1.34 \pm 0.17$ & $1.24 \pm 0.17$ \\
\hline \multicolumn{3}{|l|}{ Bright spotty lesion } \\
\hline Negative & $1(7 \%)$ & $18(69 \%)$ \\
\hline Positive & $14(93 \%)$ & $8(31 \%)$ \\
\hline \multicolumn{3}{|l|}{ Involved segment } \\
\hline Cervical & $5(33 \%)$ & $7(27 \%)$ \\
\hline Cervicothoracic & $8(53 \%)$ & $5(19 \%)$ \\
\hline Thoracic & $2(13 \%)$ & $10(38 \%)$ \\
\hline Thoracolumbar & $0(0 \%)$ & $1(4 \%)$ \\
\hline Holocord & $0(0 \%)$ & $3(12 \%)$ \\
\hline No. of segments involved (mean) & $9 \pm 4$ & $7 \pm 4$ \\
\hline \multicolumn{3}{|l|}{ Axial distribution } \\
\hline Central & $10(67 \%)$ & $21(81 \%)$ \\
\hline Peripheral & $4(27 \%)$ & $4(15 \%)$ \\
\hline Both & $1(7 \%)$ & $1(4 \%)$ \\
\hline$>$ Half of the cord area & $12(80 \%)$ & $24(92 \%)$ \\
\hline \multicolumn{3}{|l|}{ Enhancement } \\
\hline No enhancement & $4(27 \%)$ & $6(23 \%)$ \\
\hline$<50 \%$ enhancement & $9(60 \%)$ & $17(65 \%)$ \\
\hline$>50 \%$ enhancement & $0(0 \%)$ & $0(0 \%)$ \\
\hline Not available & $2(13 \%)$ & $3(12 \%)$ \\
\hline
\end{tabular}

${ }^{a}$ Data are number and percentage unless otherwise indicated.

${ }^{\mathrm{b}}$ Cord expansion ratio was measured only for 32 patients.

${ }^{c}$ Cord expansion ratio was measured only for 22 patients.

Table 3: Univariable logistic regression analysis

\begin{tabular}{|c|c|c|}
\hline Variable & OR $(95 \% \mathrm{CI})$ & $P$ Value \\
\hline \multicolumn{3}{|l|}{ Sex } \\
\hline Male & Reference & \\
\hline Female & $3.3(1.5-5.1)$ & $<.001$ \\
\hline \multicolumn{3}{|c|}{ Cervicomedullary involvement } \\
\hline Absent & Reference & \\
\hline Present & $1.6(0.2-3.0)$ & .02 \\
\hline \multicolumn{3}{|l|}{ Bright spotty lesion } \\
\hline Negative & Reference & \\
\hline Positive & $3.4(1.3-5.6)$ & .002 \\
\hline \multicolumn{3}{|l|}{ Cord expansion ratio } \\
\hline$\leq 1.4$ & Reference & \\
\hline$>1.4$ & $2.1(0.5-3.7)$ & .01 \\
\hline
\end{tabular}

spotty lesions on T2WI were significantly associated with AQP4 antibody seropositivity (Table 3). Age, the number of involved segments, the presence of skipped lesions, the enhancement pattern, and the axial distribution pattern of $>50 \%$ involvement in the axial plane were not significantly associated with AQP4 antibody status.

A scoring system was developed on the basis of the above results (Table 4). Equal scores were assigned for parameters associated with AQP4 antibody seropositivity, except for cervicomedullary junction involvement. Additional points were given if the lesion involved the medulla oblongata. The area under the receiver operating curve for the scoring system was 91.7 (95\% confidence interval, 82.9-100.0). With a score threshold of $\geq 3$, the sensitivity of the scoring system was $73.3 \%(11 / 15)$ and the specificity was $96.2 \%$ (25/26). The sensitivity and specificity of each parameter are as follows: female sex (sensitivity, $87 \%$; specificity, $81 \%$ ), cervicomedullary junction involvement (sensitivity, $60 \%$; specificity, $77 \%$ ), cord expansion ratio $>1.4$ (sensitivity, $40 \%$; specificity, $82 \%$ ), and bright spotty lesions (sensitivity, 93\%; specificity, 69\%). The interclass correlation value for measuring the cord expansion ratio was 0.78 (95\% confidence interval, $0.61-0.88)$. The $\kappa$ value for interobserver agreement in judging bright spotty lesions was 0.61 (95\% confidence interval, 0.37-0.85).

\section{DISCUSSION}

In our study, we identified characteristic spinal MR imaging features in Asian patients with AQP4-positive LETM (ie, probable NMOSD) compared with those with AQP4-negative LETM. Cervicomedullary junction involvement, cord expansion in the acute/early stage, the presence of bright spotty lesions, and female sex were significantly associated with AQP4 seropositivity in patients with LETM.

An important factor distinguishing our study from previous work is the use of serum AQP4 antibody status, rather than disease diagnostic criteria, as a reference standard. Although criteriabased diagnosis is clinically necessary, it was not appropriate to use this approach as a reference standard in our study because the aim of our study was to predict the AQP4 antibody status for the patients with LETM with unknown AQP4 antibody status. The concept of $\mathrm{NMO}$ as a disease has recently been expanded to a spectrum of disorders (NMOSD) based on the International Panel for NMO Di- 
Table 4: Scoring system

\begin{tabular}{lc}
\multicolumn{1}{c}{ Parameters } & Points \\
\hline Sex & \\
Male & 0 \\
Female & 1 \\
Cervicomedullary involvement & 0 \\
$\quad$ Absent & 1 \\
$\quad$ Present & $+1=2$ \\
Medulla oblongata involvement & \\
Bright spotty lesion & 0 \\
$\quad$ Absent or suspicious & 1 \\
Definite & \\
Cord expansion ratio for acute or early-stage MRI & 0 \\
$\quad \leq 1.4$ & 1 \\
\hline 1.4 &
\end{tabular}

agnosis criteria, and this definition may continue to evolve in the future. Additionally, LETM is not specific for NMOSD and may be representative of other disease states that cannot be further classified using the current diagnostic criteria.

Female predilection, extension of cervical lesions to brain stem lesions, ${ }^{15-17}$ spinal MR imaging findings of cord swelling, ${ }^{14,18}$ and bright spotty lesions ${ }^{13}$ have previously been reported in patients with NMO; however, the aforementioned characteristics were mostly descriptive data without statistical inference. Although Yonezu et $\mathrm{al}^{13}$ reported bright spotty lesions as statistically discriminative findings with a sensitivity and specificity of $54 \%$ and 97\%, the comparison was done within patient groups with NMO and MS. ${ }^{13}$ Recently, Pekcevik et $\mathrm{al}^{10}$ reported that only bright spotty lesions on T2WI are highly specific findings that can be used to differentiate NMO from other etiologies that could manifest as LETM. In contrast to a previous study by Pekcevik et al, ${ }^{10}$ we found that cervicomedullary junction involvement and a high cord expansion ratio in the acute/early stage of disease were statistically associated with AQP4 antibody seropositivity. This discrepancy might be attributed to several factors. First, the study of Pekcevik et al excluded idiopathic acute transverse myelitis or possible AQP4-negative NMOSD, which would be most cases of the AQP4-negative group in our study. Second, cord swelling is a subjective judgment of the radiologist, and few studies have addressed the degree of cord swelling. In contrast, we quantitatively measured cord swelling and showed good interobserver agreement when measurements were performed by 2 independent radiologists. Third, we have defined cervicomedullary junction involvement, a broader concept than brain stem extension, and suggested a clear margin for judging the presence of cervicomedullary junction involvement. Last, our study excluded potential cases of spinal dural arteriovenous fistula and spinal infarction, which could be diagnosed on the basis of MR imaging findings and the clinical setting.

Our study provides confirmation that bright spotty lesions on axial T2WI are highly specific for AQP4 antibody-positive LETM, in keeping with a previous study. ${ }^{10}$ The interobserver agreement for the judgment of bright spotty lesions was substantial $(\kappa=$ $0.61)^{19}$ and was higher than the minimal $\kappa$ value of 0.55 suggested for classification systems. ${ }^{20}$ Therefore, our scoring system (sensitivity, 73.3\%; specificity, 96.2\%) provides an accurate and reliable tool for diagnosing AQP4 antibody-positive LETM and possible NMOSD. The presence or history of optic neuritis was more fre- quent in the AQP4 antibody-positive group. It did not reach statistical significance $(P$ value $=.051)$ and was not incorporated in our scoring system, though it showed potential as a discriminative feature of the AQP4 antibody-positive patient group. However, the interpretation should be made with caution because the reported sensitivity and specificity of the AQP4 antibody for NMO are $58 \%-76 \%$ and $85 \%-99 \%$; thus, a positive AQP4 antibody result does not necessarily indicate NMO. ${ }^{1,21-23}$

The present study had several limitations mainly related to its retrospective design and small sample size. First, we were unable to prepare a validation set for the scoring system. Additionally, we were unable to perform a multivariable logistic regression analysis due to our sample size. Therefore, in building the scoring system, we could only assign equal scores for each parameter. Second, our study population was exclusively of Asian descent. Future studies are necessary to determine the generalizability of our findings to other populations. Third, the influence of different protocols, such as section thickness, on the presence of bright spotty lesions on axial T2WI is unknown. The use of thin-section MR imaging (which is less influenced by partial averaging effects in the craniocaudal direction) might increase the sensitivity for judging the presence of bright spotty lesions. Fourth, our study only included adult patients; therefore, our study results should not be extended for usage in the pediatric population, for which a differential diagnosis of acute disseminated encephalomyelitis with NMOSD might be important.

\section{CONCLUSIONS}

Cervicomedullary junction involvement, a higher cord expansion ratio, bright spotty lesions, and female sex are highly indicative of AQP4 antibody seropositivity in patients with LETM and may predict a diagnosis of NMOSD.

\section{REFERENCES}

1. Wingerchuk DM, Lennon VA, Pittock SJ, et al. Revised diagnostic criteria for neuromyelitis optica. Neurology 2006;66:1485-89 CrossRef Medline

2. Wingerchuk DM, Lennon VA, Lucchinetti CF, et al. The spectrum of neuromyelitis optica. Lancet Neurol 2007;6:805-15 CrossRef Medline

3. Jarius S, Wildemann B. Aquaporin-4 antibodies (NMO-IgG) as a serological marker of neuromyelitis optica: a critical review of the literature. Brain Pathol 2013;23:661-83 CrossRef Medline

4. Wingerchuk DM, Banwell B, Bennett JL, et al; International Panel for NMO Diagnosis. International consensus diagnostic criteria for neuromyelitis optica spectrum disorders. Neurology 2015;85: 177-89 CrossRef Medline

5. Cree BA, Lamb S, Morgan K, et al. An open label study of the effects of rituximab in neuromyelitis optica. Neurology 2005;64:1270-72 CrossRef Medline

6. Aguilera AJ, Carlow TJ, Smith KJ, et al. Lymphocytaplasmapheresis in Devic's syndrome. Transfusion 1985;25:54-56 CrossRef Medline

7. Weinshenker BG, O'Brien PC, Petterson TM, et al. A randomized trial of plasma exchange in acute central nervous system inflammatory demyelinating disease. Ann Neurol 1999;46:878-86 Medline

8. Keegan M, Pineda AA, McClelland RL, et al. Plasma exchange for severe attacks of CNS demyelination: predictors of response. Neurology 2002;58:143-46 CrossRef Medline

9. Nozaki I, Hamaguchi T, Komai K, et al. Fulminant Devic disease successfully treated by lymphocytapheresis. J Neurol Neurosurg Psychiatry 2006;77:1094-95 Medline 
10. Pekcevik Y, Mitchell CH, Mealy MA, et al. Differentiating neuromyelitis optica from other causes of longitudinally extensive transverse myelitis on spinal magnetic resonance imaging. Mult Scler 2016;22:302-11 CrossRef Medline

11. Waters $\mathrm{P}$, Jarius S, Littleton E, et al. Aquaporin-4 antibodies in neuromyelitis optica and longitudinally extensive transverse myelitis. Arch Neurol 2008;65:913-19 Medline

12. Cassinotto C, Deramond H, Olindo S, et al. MRI of the spinal cord in neuromyelitis optica and recurrent longitudinal extensive myelitis. J Neuroradiol 2009;36:199-205 CrossRef Medline

13. Yonezu T, Ito S, Mori M, et al. "Bright spotty lesions" on spinal magnetic resonance imaging differentiate neuromyelitis optica from multiple sclerosis. Mult Scler 2014;20:331-37 CrossRef Medline

14. Kitley JL, Leite MI, George JS, et al. The differential diagnosis of longitudinally extensive transverse myelitis. Mult Scler 2012;18: 271-85 CrossRef Medline

15. Wingerchuk DM, Hogancamp WF, O'Brien PC, et al. The clinical course of neuromyelitis optica (Devic's syndrome). Neurology 1999; 53:1107-14 CrossRef Medline

16. Asgari N, Skejoe HP, Lillevang ST, et al. Modifications of longitudinally extensive transverse myelitis and brainstem lesions in the course of neuromyelitis optica (NMO): a population-based, descriptive study. BMC Neurol 2013;13:33 CrossRef Medline

17. Kim W, Park MS, Lee SH, et al. Characteristic brain magnetic resonance imaging abnormalities in central nervous system aquaporin-4 autoimmunity. Mult Scler 2010;16:1229-36 CrossRef Medline

18. Qiu W, Wu JS, Zhang MN, et al. Longitudinally extensive myelopathy in Caucasians: a West Australian study of $\mathbf{2 6}$ cases from the Perth Demyelinating Diseases Database. J Neurol Neurosurg Psychiatry 2010;81:209-12 CrossRef Medline

19. Landis JR, Koch GG. The measurement of observer agreement for categorical data. Biometrics 1977;33:159-74 CrossRef Medline

20. Sanders RW. The problem with apples and oranges. J Orthop Trauma 1997;11:465-66 CrossRef

21. Lennon VA, Wingerchuk DM, Kryzer TJ, et al. A serum autoantibody marker of neuromyelitis optica: distinction from multiple sclerosis. Lancet 2004;364:2106-12 CrossRef Medline

22. Jarius S, Franciotta D, Bergamaschi R, et al. NMO-IgG in the diagnosis of neuromyelitis optica. Neurology 2007;68:1076-77 CrossRef Medline

23. Nakashima I, Fujihara K, Miyazawa I, et al. Clinical and MRI features of Japanese patients with multiple sclerosis positive for NMO-IgG. J Neurol Neurosurg Psychiatry 2006;77:1073-75 CrossRef Medline 